\title{
Synthesis of Some New Bispyrazolidine Derivatives for Biological Interest
}

\author{
Eman F. Kirolos ${ }^{1}$, Amal S. Yanni2 ${ }^{*}$ \\ ${ }^{1}$ College of Pharmacy, University of Florida, USA \\ ${ }^{2}$ Chemistry Department, Faculty of Science, Assiut University, Assiut, Egypt \\ Email: e.kirolos@ufl.edu, ^profayanni@yahoo.com
}

How to cite this paper: Kirolos, E.F. and Yanni, A.S. (2020) Synthesis of Some New Bispyrazolidine Derivatives for Biological Interest. International Journal of Organic Chemistry, 10, 104-110.

https://doi.org/10.4236/ijoc.2020.102007

Received: May 6, 2020

Accepted: June 27, 2020

Published: June 30, 2020

Copyright $\odot 2020$ by author(s) and Scientific Research Publishing Inc. This work is licensed under the Creative Commons Attribution International License (CC BY 4.0).

http://creativecommons.org/licenses/by/4.0/

\begin{abstract}
One pot criss-cross cycloaddition reaction of substituted 2,3-diaz-1,3-butadienes (1) and/or (2) with two equivalents of 2,3-dichloro-1,4-naphthoquinone in presence of dry benzene afforded bisnaphth[2,3-c]pyrazolidinetetrone derivatives (3) \& (4). The chemical structure was achieved by elemental and spectral analysis. Biological activity of the synthesized compounds was tested against some micro-organisms, and has shown an interesting fungicidal activity and strongly to remarkable bactericidal activity.
\end{abstract}

\section{Keywords}

Bispyrazolidine, Cycloaddition, Antimicrobial Activity

\section{Introduction}

Tricyclic pyrazole derivatives were described as inhibitor of growth of Bacillus subtilis, Pseudomonas fluorescens, Staphylococcus aureus and KB cells at moderate concentrations [1]. Many of 4-alkyl and 4,4'-dialkyl-1,2-bis(4-chlorophenyl) pyrazolidine-3,5-dione derivatives demonstrated good activity against MurbB in vitro and low MIC values against Gram-positive bacteria, particularly penicillin-resistant streptococcus pneumoniae (PRSP) [2]. Moreover, 4,4'-(arylmethylen) bis $(1 \mathrm{H}$ pyrazol-5-ols), were evaluated for in vitro antiviral activity against peste des petits ruminants virus (PPRV). One of these series emerged at the most interesting compound exhibiting excellent antiviral activity against PPRV and was found to be more potent than the standard drug [3]. Asma Samaunnisa [4] also reported that a set of novel of 2,6-dimethyl-1,4-dihydopyridine-3,5-yl-bis[carbonyl-2-(phenyl)] pyrazolidine-3,5-diones], were subjected to in vivo anti-inflammatory and analgesic activities. Activity of all the derivatives was compared with the chosen standards namely indomethacin and tramadol for anti-inflammatory and analgesic activity 
respectively using dimethylsulfoxide as control. Almost all the derivatives exhibited significant activity. Moreover, the above compounds [5] were also subjected to anticonvulsant and sedative activities screening. Methods: Anticonvulsant activity was tested using two models namely Maximal electroshock (MES) induced convulsion method and Pentylenetetrazole (PTZ) induced convulsion method. Sedative activity was also screened with two models viz. locomotor activity using actophotometer in mice and anxiolytic activity using the elevated plus maze in rats. Phenytoin, diazepam and chlorpromazine, diazepam were used as standards for anticonvulsant and sedative activities respectively. Results: At both the doses of $200 \mathrm{mg} / \mathrm{kg}$ and $400 \mathrm{mg} / \mathrm{kg}$, all the tested derivatives exhibited significant anticonvulsant activities. Conversely, none of the derivatives exhibited better sedative activity which can be compared with the standard. In view of these observations, the intention was directed to synthesize some new bispyrazolidine derivatives of expected biological interest.

\section{Experimental}

All melting points are uncorrected. IR spectra were recorded (KBr) with a Perkin-Elmer 1430 spectrophotometer. ${ }^{1} \mathrm{H}$ NMR spectra were obtained on Varian EM $399.65 \mathrm{MHz}$ equipment. MS spectra were recorded with a Jeol the MS route JMS.600 H.

\subsection{Preparation of Substituted 2,3-Diaza-1,3-butadienes (1) and/or (2)}

Aromatic azines were prepared according to the known procedures by condensation of aromatic aldehydes or acetophenone and hydrazine sulphate.

\subsection{Preparation of Bisnaphth[2,3-c]pyrazolidinetetrone (3a-f) and (4)}

\section{General procedure:}

A mixture of aldazine or acetophenonazine (1 and/or $2,1 \mathrm{mmole})$ and 2,3-dichloronaphthoquinone ( $2 \mathrm{mmole})$ was heated to $100^{\circ} \mathrm{C}$ for few hours and then refluxed in $10 \mathrm{ml}$ dry benzene for $10-13 \mathrm{hrs}$. The solution was concentrated near dryness and diluted with methanol and the separated products were filtered off and crystallized from ethanol or chloroform. Yield 45\% - 65\%.

\subsubsection{Adduct (3a), $\mathrm{R}=-\mathrm{C}_{6} \mathrm{H}_{5}: 5^{\prime}, 7^{\prime}, 13^{\prime}, 15^{\prime}$-Tetrachloro-6,14-diphenyl- bisnaphth[2,3-c;2,3-g]perhydropyrazolo[1,2-a]pyrazole- 5,8,13,16-tetrone}

Red substance, m.p. $197^{\circ} \mathrm{C}$, obtained from benzalazine and 2,3-dichloronaphthoquinone in 52\% yield. Anal Calcd. For $\mathrm{C}_{34} \mathrm{H}_{20} \mathrm{Cl}_{4} \mathrm{~N}_{2} \mathrm{O}_{4}$ (662.32): C, 61.65; H, 3.04; $\mathrm{Cl}, 21.41 ; \mathrm{N}, 4.23$. Found: $\mathrm{C}, 61.90 ; \mathrm{H}, 3.24 ; \mathrm{Cl}, 21.65 ; \mathrm{N}, 4.36$. IR $\left(\mathrm{cm}^{-1}\right): v$ $=1670(>\mathrm{C}=\mathrm{O}) ; 1270(\mathrm{C}-\mathrm{N}) ; 740(\mathrm{C}-\mathrm{Cl}) .{ }^{1} \mathrm{H}$ NMR $\left(\mathrm{CDCl}_{3}\right): \delta=4.7(\mathrm{~s}, 2 \mathrm{H}$, $2 \mathrm{CH}-\mathrm{N}), 7.24-8.3(\mathrm{~m}, 18 \mathrm{H}, \mathrm{Ar}-\mathrm{H}) . \mathrm{MS} \mathrm{m} / \mathrm{z}(\%):\left[\mathrm{M}^{+}-4 \mathrm{HCl}-2 \mathrm{C}_{6} \mathrm{H}_{5}-2 \mathrm{H}\right] 359$ $(0.2)$. 
2.2.2. Adduct (3b), $\mathrm{R}=-\mathrm{C}_{6} \mathrm{H}_{4}-p-\mathrm{OCH}_{3}: 5^{\prime}, 7^{\prime}, 13^{\prime}, 1^{\prime}$ '-Tetrachloro-6,14-di( $p$-methoxyphenyl)bisnaphth[2,3-c;2,3-g]perhydropyrazolo-[1,2-a] pyrazole-5,8,13,16-tetrone

Violet brown substance, m.p. $160^{\circ} \mathrm{C}$, obtained from anisalazine and 2,3-dichloronaphthoquinone in 65\%. Anal Calcd. For $\mathrm{C}_{36} \mathrm{H}_{24} \mathrm{Cl}_{4} \mathrm{~N}_{2} \mathrm{O}_{6}$ (722.36): C, 59.85; $\mathrm{H}$, 3.35; Cl, 19.63; N, 3.91. Found: C, 60.15; H, 3.50; Cl, 19.75; N, 3.94. IR $\left(\mathrm{cm}^{-1}\right): v=$ $1680(>\mathrm{C}=\mathrm{O}) ; 1440\left(\mathrm{C}-\mathrm{H}\right.$ bending of $\left.\mathrm{CH}_{3}\right), 2880$ (w-CH stretching of $\left.\mathrm{CH}_{3}\right) ; 1270$ $(\mathrm{C}-\mathrm{N}) ; 700(\mathrm{C}-\mathrm{Cl}) .{ }^{1} \mathrm{H}$ NMR $\left(\mathrm{CDCl}_{3}\right): \delta=3.9\left(\mathrm{~s}, 6 \mathrm{H}, 2-\mathrm{OCH}_{3}\right), 4.5(\mathrm{~s}, 2 \mathrm{H}, 2 \mathrm{CH}-\mathrm{N})$, 7 - $8.2(\mathrm{~m}, 16 \mathrm{H}, \mathrm{Ar}-\mathrm{H})$. MS m/z (\%): [ $\left.\mathrm{M}^{+}-2 \mathrm{C}_{6} \mathrm{H}_{4}-\mathrm{OCH}_{3}-\mathrm{C}_{10} \mathrm{H}_{4} \mathrm{O}_{2} \mathrm{Cl}_{2}-5 \mathrm{H}\right] 276$ (11.2).

2.2.3. Adduct (3c), R = - $\mathrm{C}_{6} \mathrm{H}_{4}-0-0 \mathrm{H}^{\prime} 5^{\prime}, 7^{\prime}, 13^{\prime}, 15^{\prime}-\mathrm{Tetrachloro}^{-6,14-}$ $\operatorname{di}(o-h y d r o x y p h e n y l) b i s n a p h t h[2,3-c ; 2,3-g] p e r h y d r o p y r a z o l o[1,2-a]$ pyrazole-5,8,13,16-tetrone

Brown red substance, m.p. $170^{\circ} \mathrm{C}$, obtained from $o$-hydroxy-benzalazine and 2,3-dichloronaphthoquinone in $45 \%$ yield. Anal Calcd. For $\mathrm{C}_{34} \mathrm{H}_{20} \mathrm{Cl}_{4} \mathrm{~N}_{2} \mathrm{O}_{6}$ (694.30): C, 58.81; H, 2.90; Cl, 20.42; N, 4.03. Found: C, 58.99; H, 3.0; Cl, 20.29; $\mathrm{N}, 4.20$. IR $\left(\mathrm{cm}^{-1}\right): v=1670(>\mathrm{C}=\mathrm{O}) ; 1270(\mathrm{C}-\mathrm{N}) ; 720(\mathrm{C}-\mathrm{Cl}) ; 3480(-\mathrm{OH}) .{ }^{1} \mathrm{H}$ NMR (DMSO): $\delta=4.5(\mathrm{~s}, 2 \mathrm{H}, 2 \mathrm{CH}-\mathrm{N}), 7.0-8.2(\mathrm{~m}, 16 \mathrm{H}, \mathrm{Ar}-\mathrm{H}), 9.0$ (s, 2H, 2OH). MS $m / z(\%):\left[\mathrm{M}^{+}-\mathrm{H}_{2} \mathrm{O}-\mathrm{C}_{6} \mathrm{H}_{5}-\mathrm{HCl}\right] 562$ (2.4).

\subsubsection{Adduct (3d), $\mathrm{R}=-\mathrm{C}_{6} \mathrm{H}_{4}-p-\mathrm{Cl}: 5^{\prime}, 7^{\prime}, 13^{\prime}, 15^{\prime}$-Tetrachloro-6,14- di(p-chlorophenyl)bisnaphth[2,3-c;2,3-g]perhydropyrazolo[1,2-a] pyrazole-5,8,13,16-tetrone}

Brown red substance, m.p. $220^{\circ} \mathrm{C}$, obtained from $p$-chloro-benzalazine and 2,3-dichloronaphthoquinone in $49 \%$ yield. Anal Calcd. For $\mathrm{C}_{34} \mathrm{H}_{18} \mathrm{Cl}_{6} \mathrm{~N}_{2} \mathrm{O}_{4}$ (731.20): C, 55.84; H, 2.48; Cl, 29.08; N, 3.83. Found: C, 56.05; H, 2.60; Cl, 28.99; $\mathrm{N}$, 3.97. IR $\left(\mathrm{cm}^{-1}\right): v=1680(>\mathrm{C}=\mathrm{O}) ; 1270(\mathrm{C}-\mathrm{N}) ; 730(\mathrm{C}-\mathrm{Cl}) . \mathrm{MS} \mathrm{m} / z(\%)$ : $\left[\mathrm{M}^{+}-4 \mathrm{O}-\mathrm{H}^{+}\right] 666.1(0.4)$.

\subsubsection{Adduct (3e), $\mathrm{R}=-\mathrm{CH}=\mathrm{CH}-\mathrm{C}_{6} \mathrm{H}_{5}: 5^{\prime}, 7^{\prime}, 13^{\prime}, 15^{\prime}-$ Tetrachloro-6,14-di- (2-phenylethenyl)bisnaphth[2,3-c;2,3-g]perhydropyrazolo[1,2-a] pyrazole-5,8,13,16-tetrone}

Light green substance, m.p. $185^{\circ} \mathrm{C}$, obtained from cinnamaldazine and 2,3-dichloronaphthoquinone in $45 \%$ yield. Anal Calcd. For $\mathrm{C}_{38} \mathrm{H}_{24} \mathrm{Cl}_{4} \mathrm{~N}_{2} \mathrm{O}_{4}$ (714.40): C, 63.88; H, 3.39; Cl, 19.84; N, 3.92. Found: C, 64.14; H, 3.54; Cl, 20.0; $\mathrm{N}, 4.08$. IR $\left(\mathrm{cm}^{-1}\right): v=1670(>\mathrm{C}=\mathrm{O}) ; 1440,950$ ( $\mathrm{CH}$ bend of substituted alkene); $1270(\mathrm{C}-\mathrm{N}) ; 700$ (C-Cl). MS m/z (\%): $\left[\mathrm{M}^{+}-2 \mathrm{CH}=\mathrm{CH}-\mathrm{C}_{6} \mathrm{H}_{5}-\mathrm{C}_{10} \mathrm{H}_{4} \mathrm{O}_{2} \mathrm{Cl}_{2}-2 \mathrm{CO}^{+}\right]$ 225.8 (80.9).

\subsubsection{Adduct (3f), $\mathrm{R}=-\mathrm{C}_{4} \mathrm{H}_{3} \mathrm{O}: 5^{\prime}, 7^{\prime}, 13^{\prime}, 15^{\prime}$-Tetrachloro-6,14- di(2-furyl)-bisnaphth[2,3-c;2,3-g]perhydropyrazolo[1,2-a] pyrazole-5,8,13,16-tetrone}

Yellowish green substance, m.p. $200^{\circ} \mathrm{C}$, obtained from furfuralazine and 2,3-dichloronaphthoquinone in $40 \%$ yield. Anal Calcd. For $\mathrm{C}_{30} \mathrm{H}_{16} \mathrm{Cl}_{4} \mathrm{~N}_{2} \mathrm{O}_{6}$ (642.22): C, 56.10; H, 2.51; Cl, 22.08; N, 4.36. Found: C, 56.32; H, 2.68; Cl, 22.28; $\mathrm{N}, 4.50$. IR $\left(\mathrm{cm}^{-1}\right): v=1675(>\mathrm{C}=\mathrm{O}) ; 1270(\mathrm{C}-\mathrm{N}) ; 1180-1130$ (-C-O-C cyclic); 700 (C-Cl). ${ }^{1} \mathrm{H} \mathrm{NMR}\left(\mathrm{CDCl}_{3}\right): \delta=4.7$ (s, 2H, 2CH-N), $7.2-8.18$ (m, 14H, Ar-H). 
MS m/z (\%): $\left[\mathrm{M}^{+}-2 \mathrm{C}_{4} \mathrm{H}_{3} \mathrm{O}-\mathrm{C}_{10} \mathrm{H}_{4} \mathrm{O}-\mathrm{Cl}\right] 316.5$ (5.8).

\subsubsection{Adduct (4): 5',7',13',15'-Tetrachloro-6,14-di(methyl, phenyl)-bis-naphth[2,3-c;2,3-g]pyrazolol[1,2-a]pyrazole- 5,8,13,16-tetrone}

Green substance, m.p. $190^{\circ} \mathrm{C}$, obtained from azine of acetophenone and 2,3-dichloronaphthoquinone in $40 \%$ yield. Anal Calcd. For $\mathrm{C}_{36} \mathrm{H}_{24} \mathrm{Cl}_{4} \mathrm{~N}_{2} \mathrm{O}_{4}$ (690.38): C, 62.63; H, 3.51; Cl, 20.53; N, 4.06. Found: C, 62.85; H, 3.65; Cl, 20.40; $\mathrm{N}$, 3.96. IR $\left(\mathrm{cm}^{-1}\right): v=1670(>\mathrm{C}=\mathrm{O}) ; 1270(\mathrm{C}-\mathrm{N}) ; 1400\left(\mathrm{CH}\right.$ bending of $\left.\mathrm{CH}_{3}\right)$; 720 (C-Cl). ${ }^{1} \mathrm{H}$ NMR (TFA): $\delta=1.3$ (s, $6 \mathrm{H}, 2 \mathrm{CH}_{3}$ ), $8.0-8.3$ (m, 18H, Ar-H). MS $\mathrm{m} / \mathrm{z}(\%):\left[\mathrm{M}^{+}-\mathrm{C}_{6} \mathrm{H}_{5}-2 \mathrm{CH}_{3}+3 \mathrm{H}^{+}\right] 586.5$ (2.6).

\subsection{Antimicrobial Activity}

The fungal species were grown in sterilized $9.0 \mathrm{~cm}$ Petri dishes containing potato's dextrose agar (PDA) supplemented with $0.05 \%$ chloramphenicol to suppress bacterial contamination (Al-Doory, 1980). From these culture, agar discs (10 $\mathrm{mm}$ diameter) containing spores and hyphae were transferred aseptically to screw topped vials containing $20 \mathrm{ml}$ sterile distilled water. After thorough shaking $1 \mathrm{ml}$ samples of the spores suspension were pipetted into sterile Petri dishes, followed by the addition of $15 \mathrm{ml}$ liquefied PDA medium was then left to solidify. The tested compounds were dissolved in ethylene glycol to give $2.0 \%$ concentration. Antifungal activity was determined according to the method by Bauer et al. (1966) [6] using $3 \mathrm{~mm}$ diameter filter paper discs (Watmann No. 3) loaded with $10 \mu \mathrm{L}$ of the solution under investigation (2.0\%). The discs were placed on the surface of the fungal cultures which were incubated at $30^{\circ} \mathrm{C}$. The diameter of the inhibition zone around each disc was measured (c.f. Table 1). The previous method was used for determining antibacterial activity [7] (c.f. Table 2).

\section{Results and Discussion}

A cyclic diene system containing $\mathrm{N}$ or $\mathrm{O}$ does not appear to undergo normal Diels Alder reactions [8]. Wagner [9] found that heating one mole of benzalazine with 2 moles of maleic anhydride to $100^{\circ} \mathrm{C}$ in dry benzene for several hours gave an addition compound through simultaneous 1,3 and 2,4 addition of 2 moles of

Table 1. Fungicidal activity is expressed as inhibition zone in $\mathrm{mm}$ of compounds (3a-f) and (4).

\begin{tabular}{cccccc}
\hline Compd. & Organism & & & \\
& & Asp. fumigatus & Asp. niger & Asp. flavous & Candida albicans \\
\hline 3a & 12 & 33 & 14 & 10 \\
3b & 12 & 25 & 23 & 9 \\
3c & 15 & 33 & 25 & 10 \\
3d & 24 & 33 & 22 & 10 \\
3e & 12 & 30 & 19 & - \\
3f & 10 & 28 & 12 & 10 \\
4 & 24 & 22 & 20 & \\
\hline
\end{tabular}


Table 2. Bactericidal activity is expressed as inhibition zone in $\mathrm{mm}$.

\begin{tabular}{|c|c|c|c|c|}
\hline Compd. & M. luteus & M. roses & B. cereus & Staph. aureus \\
\hline $3 a$ & 15 & 10 & 2 & 8 \\
\hline $3 b$ & 22 & 15 & 11 & - \\
\hline $3 c$ & 20 & 8 & 10 & 9 \\
\hline $3 d$ & 17 & 13 & 11 & - \\
\hline $3 e$ & 9 & 11 & 10 & 10 \\
\hline $3 f$ & 9 & 12 & 11 & 10 \\
\hline 4 & 31 & 10 & 12 & - \\
\hline
\end{tabular}

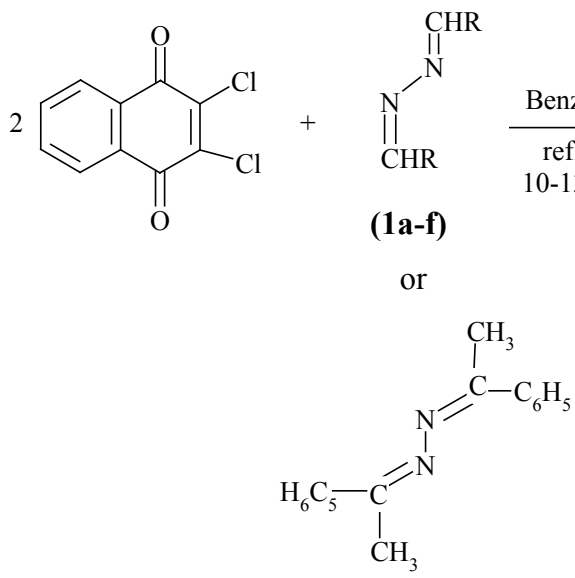

(2)

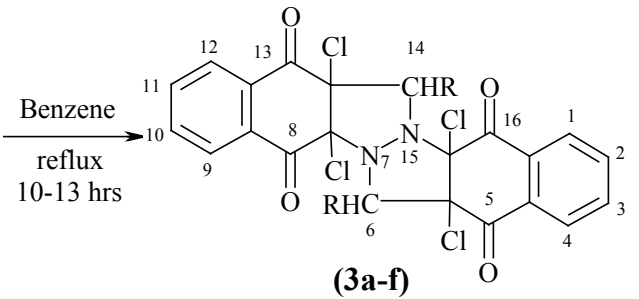

(3a-f)

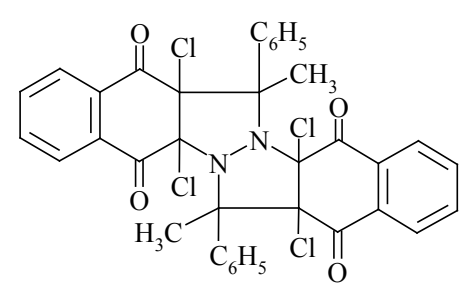

(4)<smiles>[R][CH]N1N(C)C([R])C2(Cl)C(=O)c3ccccc3C(=O)C12Cl</smiles>

(5)

Scheme 1. $\mathrm{R}=-\mathrm{C}_{6} \mathrm{H}_{5}(1 \mathrm{a}, 3 \mathrm{a}) ;-\mathrm{C}_{6} \mathrm{H}_{4}-p-\mathrm{OCH}_{3}(\mathbf{1 b}, 3 \mathrm{~b}) ;-\mathrm{C}_{6} \mathrm{H}_{4}-o-\mathrm{OH}(1 \mathrm{c}, 3 \mathrm{c}) ;-\mathrm{C}_{6} \mathrm{H}_{4}-p-\mathrm{Cl}$ (1d, 3d); $-\mathrm{CH}=\mathrm{CH}-\mathrm{C}_{6} \mathrm{H}_{5}(1 \mathrm{e}, 3 \mathrm{e}) ;-\mathrm{C}_{4} \mathrm{H}_{3} \mathrm{O}$ (1f, 3f).

maleic anhydride to benzalazine which was designated as criss-cross addition. Moreover, aromatic 1,4-disubstituted-1,4-diazbuta-1,3-dienes with thiocyanates in glacial acetic acid via criss-cross cycloaddition produced the corresponding perhydroimidazo[4,5]imidazole-2,5-dithione [10]. Similarly and continuation to my previous work [11] [12] [13] [14] [15], aldazines (1a-f) such as benzalazine, anisalazine, $o$-hydroxybenzalazine, $p$-chloro-benzalazine, cinnamaldazine, furfuralazine and/or acetophenonazine (2) were allowed to react with 2 moles of 2,3-dichloronaphtho-1,4-quinone in dry benzene and afforded bispyrazolidines (3a-f) and (4) (Scheme 1).

Huisgen [16] suggested that criss-cross reaction can best be represented by series of two [3+2]-cycloaddition steps. Azomethine imine (5) was postulated as a 
key intermediate [8]. The compounds produced satisfactory results for elemental and spectral analysis.

As for their fungicidal activity, all compounds (3d) and (4) were more active against Asp. fummigatus than others, whereas compounds (3b, c, d) and (4) had a greater activity against Asp. flavous.

And for their bactericidal activity, while most compounds exhibited a remarkable bactericidal activity against $B$. cereus and $M$. roses, compounds $(3 \mathrm{~b}, \mathrm{c})$ and (4) had a strong activity against $M$. luteus and compounds (3a, c, e, f) were active against $S$. aureus.

\section{Conclusion}

The title compounds are synthesized successfully through simple novel and one pot reaction from substituted 2,3-diaza-1,3-butadienes and 2,3-dichloro-1,4naphthoquinone. These compounds also exhibited an interesting fungicidal activity and strongly to remarkable bactericidal activity against some tested micro-organisms.

\section{Conflicts of Interest}

The authors declare no conflicts of interest regarding the publication of this paper.

\section{References}

[1] Hashem, M.M., Berlin, K.D., Chessnut, R.W. and Durham, N.N. (1976) Novel Pyrazolo and Thiazolo Steroidal Systems and Model Analogs Containing Dimethoxylaryl (or Dihydroxylazyl) Groups and Derivatives Synthesis, Spectral Properteis and Biolgoical Activity. Journal of Medicinal Chemistry, 19, 229.

https://doi.org/10.1021/jm00224a007

[2] Kitterer, K.M.K., Davis, J.M., et al. (2005) 4-Alkyl and 4,4'-Dialkyl-1,2-bis(4Chlorophenyl)pyrazolidine-3,5-dione Derivatives as New Inhibitors of Bacterial Cell Wall Biosynthesis. Bioorganic and Medicinal Chemistry Letters, 15, 2527-2531. https://doi.org/10.1016/j.bmcl.2005.03.058

[3] Sujatha, K., Shanthi, G., et al. (2009) Synthesis and Antiviral Activity of 4,4'-(Arylmethylene)bis(1H-pyrazole-5-ols) against Peste De Petits Ruminant Virus (PPRV). Bioorganic and Meidicinal Chemistry Letters, 19, 4501-4503. https://doi.org/10.1016/j.bmcl.2009.02.113

[4] Asma Samaunnisa, A., Mohammed, R. and Madhavan, V. (2013) In-Vivo Anti-Inflammatory and Analgesic Activities of Novel Derivatives of Bispyrazolidine-3,5-dione Tethered with 1,4-Dihydropyridine Moiety. International Journal of Research in Pharmacy and Chemistry, 3, 813-818.

[5] Asma Samaunnisa, A., Mohammed, R., Venkataramana, C.H.S. and Madhavan, V. (2014) Assessment of Anticonvulsant and Sedative Activities of Bispyrazolidine-3,5-dione Derivatives Tethered with 1,4-Dihydropyridine Moiety. International Journal of Pharmacy and Pharmaceutical Sciences, 6, 601-605. https://doi.org/10.7897/2277-4343.05123

[6] Bauer, A.W., Kibry, M.M. and Truck, J.C.M. (1966) Antibiotic Susceptibility Testing by a Standardized Single Disk Method. American Journal of Clinical Pathology, 
45, 493-496. https://doi.org/10.1093/ajcp/45.4_ts.493

[7] Mahfouz, N.M. and Moharram, A.M. (1999) Synthesis, Characterization and In-Vitro Antifungal Evaluation of Some Dithiocarbamide Acid Derivatives. Pharmacol. Commun., 5, 315.

[8] Van Alphen, J. (1942) The Reaction of Azines with Maleic Acid Anydride. Recueil des Travaux Chimiques des Pays-Bas, 61, 892-895. https://doi.org/10.1002/recl.19420611211

[9] Wagner-Jauregg, T. (1930) Uber Addierende Heteropolymerisation. Chemische Berichte, 63, 3218-3224. https://doi.org/10.1002/cber.19300631140

[10] Verner, J. and Potacek, M. (2004) Aromatic Glyoxalimines in Criss-Cross Cycloaddition. Central European Journal of Chemistry, 2, 220-233. https://doi.org/10.2478/BF02476192

[11] Yanni, A.S. (2002) Synthesis of Some New Bispyrrolidine Derivatives for Biological Interest. 6th International Conference on Physical Organic Chemistry, San Diego, 4-9 August 2002.

[12] Yanni, A.S. (2004) Synthesis of Some New Bispyrrolidine Derivatives for Biological Interest: Part II. Polymer Networks Conference, Bethesda, 15-19 August 2004.

[13] Yanni, A.S. (2006) Synthesis of Some New Bispyrrolidine Derivatives for Biological Interest: Part III. 10th Annual Green Chemistry Conference, Washington DC, 24-29 June 2006.

[14] Yanni, A.S. (2014) Synthesis of Some New Bistriazolidine Derivatives and Their Biological Activity. Russian Journal of Bioorganic Chemistry, 40, 377-378. https://doi.org/10.1134/S1068162014020150

[15] Yanni, A.S. (2016) Synthesis of Some New Bisindole Derivatives and Their Biological Activity. International Journal of Organic Chemistry, 6, 187-191. https://doi.org/10.4236/ijoc.2016.64019

[16] Huisgen, R. (1963) 1,3-Dipolar Cycloadditions: Past and Future. Angewandte Chemie, 2, 565-598. https://doi.org/10.1002/anie.196305651 